\title{
Anticancer Activity of Tetrandrine by Inducing Apoptosis in Human Breast Cancer Cell Line MDA-MB-231 In Vivo
}

\author{
Chun-hui Wang $\mathbb{D},{ }^{1}$ Jia-min Yang $\left(\mathbb{D},{ }^{2}\right.$ Yu-bo Guo $\left(\mathbb{D},{ }^{3}\right.$ Jing Shen, ${ }^{1}$ and Xiao-hua Pei $\mathbb{D}^{1}$ \\ ${ }^{1}$ The Fanshan Hospital, Beijing University of Chinese Medicine, Beijing 102488, China \\ ${ }^{2}$ Beijing Vocational College of Labour and Social Security, Beijing 100029, China \\ ${ }^{3}$ Beijing University of Chinese Medicine, Beijing 100029, China \\ Correspondence should be addressed to Xiao-hua Pei; pxh_127@163.com
}

Received 13 March 2020; Accepted 15 June 2020; Published 30 June 2020

Academic Editor: Daniela Rigano

Copyright (c) 2020 Chun-hui Wang et al. This is an open access article distributed under the Creative Commons Attribution License, which permits unrestricted use, distribution, and reproduction in any medium, provided the original work is properly cited.

\begin{abstract}
Tetrandrine (TET) is an alkaloid extracted from a traditional Chinese medicinal plant. It exerts remarkable anticancer activity and induces apoptotic cell death in various human cancer cells. The present study aimed to investigate the effects of TET on the inhibition of tumor growth and the induction of apoptosis in MDA-MB-231 breast cancer in xenograft mice. Tumor weight and volume were measured. The histopathological changes in the tumor tissue were observed. Immunohistochemistry analysis of Bcl2-associated X protein (Bax) and B-cell lymphoma/leukemia-2 (Bcl-2) was carried out. The expression of apoptosis-associated genes and proteins, such as cysteine aspartic acid-specific protease-3 (Caspase-3), Survivin, Bax, Bcl-2, BH3-interacting domain death agonist (Bid), and poly ADP-ribose polymerase (PARP), was measured by reverse transcription-polymerase chain reaction (RT-PCR) and Western blotting, respectively. TET inhibited tumor growth and induced apoptosis in TNBC cell line MDA-MB231. The mechanism underlying this effect might be mediated by TET-upregulated Caspase-3, Bax, and Bid and downregulated by Bcl-2, Survivin, and PARP. Taken together, this study supported the fact that TET is a promising therapeutic agent for the treatment of TNBC, thereby providing experimental evidence for its use in the treatment of breast cancer.
\end{abstract}

\section{Introduction}

The incidence of breast cancer accounts for $7-10 \%$ of all malignant tumors in the body [1]. It is one of the most common tumors in females that threatens women health with increasing incidence. According to the survey in 2016, 246,660 invasive breast cancer patients were detected in the USA, of which about $1 / 6$ deceased [2]. The incidence of breast cancer is also increasing every year in China. At present, the number of new breast cancer patients in China accounts for about $12.2 \%$ of the worldwide cases, and the number of deaths accounts for about $9.6 \%$ of the global rate [3].

Breast cancer is categorized into two types: noninvasive and invasive. Clinically, it is classified into three types: hormone receptor-positive, human epidermal growth factor receptor-2 (HER2) positive, and triple-negative breast cancer (TNBC) [4]. As an invasive breast carcinoma, TNBC is characterized by the absence of expression of the estrogen receptor (ER), progesterone receptor (PgR), and human epidermal growth factor receptor-2 (HER2) proteins that belong to basal cell-like breast cancer. Therefore, it was not eligible for hormone or anti-Her2 therapy. TNBC represents approximately $15-20 \%$ of all pathological types of breast cancers but accounts for a disproportionate number of breast cancer-related deaths constituting up to $5 \%$ of all cancer deaths annually $[2,5]$. As a specific subtype of breast cancer and compared to the hormone receptor-positive breast cancers, TNBC has a high recurrence rate, strong invasiveness, and a worse prognosis, which commonly occurs in younger and obese women; the average age of onset was 53 years [6]. However, targeted treatment is yet lacking. Therefore, effective prevention and cure of breast cancer, improving the survival rate and quality of life and alleviating 
the burden on patients, have become major concerns worldwide.

At present, TNBC patients undergo combination therapies, consisting of surgery, radiation, chemotherapy, newly developed targeted therapy, and immunotherapy [4]. TNBC constitutes a heterogeneous group of malignancies that differ in natural history and response to treatment. Due to the lack of targeted therapy options, the standard care for TNBC remains chemotherapy. Although TNBC is the subtype with the most complete response to chemotherapy $(22 \%)$, the recurrence and metastasis rate of such patients is still higher than that of non-TNBC tumors [6, 7]. Considering the malignancy of TNBC and the death rate of metastatic breast cancer, further studies are required to explore therapies or drugs in improving the outcome of this subtype of breast cancer.

Tetrandrine (TET) is a bis-benzylisoquinoline alkaloid isolated from a Chinese medicinal herb, han-fang-chi (or fen-fang-qi, Stephania tetrandra S. Moore) and has been used as a herbal therapy in Chinese medicine for hundreds of years. TET possesses antioxidant [8], anti-inflammatory [9], plasma glucose-lowering [10], immunosuppressive [11], antifibrotic [12], anticancer [13], antivirus [14], hypertension [15], silicosis [16], and reversal of chemotherapy drug resistance activities [17]. It also possesses remarkable antitumor activity in several types of cancers both in vitro and in vivo, including breast cancer [18, 19], liver cancer [20], colon cancer [21], leukemia [22], lung cancer [23], prostate cancer [24], and cervical cancer [25].

The primary mechanism of action of TET is related to multiple factors, such as modulating molecular signaling pathways [26, 27], inducing cancer cells apoptosis $[28,29]$, promoting cell cycle arrest [30,31], and increasing cell autophagy [18]. Although TET plays a significant role of an antibreast cancer therapeutic, its effect on TNBC is to be elucidated.

In this study, the anticancer activity of TET against the TNBC cell line MDA-MB-231 was focused on apoptosis. The histopathological changes in the tumor tissue were observed, the immunohistochemistry of $\mathrm{Bcl}-2$-associated $\mathrm{X}$ protein (Bax) and B-cell lymphoma/leukemia-2 (Bcl-2) was analyzed, and the expression of cysteine aspartic acid-specific protease-3 (Caspase-3), Survivin, Bax, Bcl-2, BH3-interacting domain death agonist (Bid), and poly ADP-ribose polymerase (PARP) genes and proteins was detected by RTPCR and Western blotting, respectively.

\section{Materials and Methods}

2.1. Animals and Grouping. A total of $30 \mathrm{female} \mathrm{BALB} / \mathrm{c}$ nude mice, aged 4 weeks weighing $16 \pm 2 \mathrm{~g}$ (purchased from Beijing Vital River Laboratory Animal Technology Co., Ltd. (Beijing, China)), were housed in a room that was maintained at a constant temperature of $23 \pm 1^{\circ} \mathrm{C}$. The room was maintained at a constant humidity of $45 p .100 \pm 5 p .100$ and a $12 \mathrm{~h}: 12 \mathrm{~h}$ light/dark cycle with light onset at 08:00 a.m. Drinking water and laboratory rodent chow were provided pour ad libitum. All studies were conducted in the facilities of the Beijing University of Chinese Medicine and approved by the Medical and Experimental Animal Ethics Committee of Beijing University of Chinese Medicine (no. BUCM-42017122028-4028).

2.2. Medicine. Capecitabine was purchased from Shanghai Roche Pharmaceutical Co., Ltd. (Shanghai, China). TET was purchased from Zhejiang Jinhua CONBA Biopharm. Co., Ltd. (Jinhua, Zhejiang, China).

2.3. Reagents and Apparatus. Mouse anti-Bax, mouse antiBcl-2, rabbit anti-Caspase-3, rabbit anti-Bid, rabbit antiPARP, rabbit anti-Survivin, and rabbit anti-GAPDH were obtained from Proteintech (USA). Horseradish peroxidase(HRP-) conjugated goat anti-mouse and anti-rabbit IgG antibodies were provided by Beijing Zhongshan Golden Bridge Biotechnology Co. Ltd. (Beijing, China). Protein assay kit was obtained from Beijing Pulilai Gene Technology Co., Ltd. (Beijing, China). Reverse transcription kit was obtained from Promega (USA).

2.4. Medicinal Dosage and Administration. The clinical dosage conversion between humans and mice was estimated based on a previous study: human medicinal dosage $(\mathrm{g} / \mathrm{kg})$ : mice medicinal dosage $(\mathrm{g} / \mathrm{kg})=12.33: 1$ [32]. If a person $(60 \mathrm{~kg})$ receives $10 \mathrm{~g}$ of each medicine, the equivalent dose for a mouse will be $2.055 \mathrm{~g} / \mathrm{kg}$. The daily dosage of intragastric administration for each nude mouse was $1 \mathrm{mg}$ for capecitabine and $2 \mathrm{mg}$ for TET. Capecitabine and TET were prepared as $10 \mathrm{mg} / \mathrm{mL}$ and $20 \mathrm{mg} / \mathrm{mL}$ suspensions in sterile deionized water, respectively.

2.5. Cell Culture. Human breast cancer cell MDA-MB-231 was obtained from Cell Resource Center, Shanghai Institutes for Biological Sciences, Chinese Academy of Sciences and cultured in Dulbecco's modified Eagle's medium (DMEM) supplemented with $10 \%$ fetal bovine serum (FBS) (Gibco, China), $100 \mathrm{U} / \mathrm{mL}$ penicillin, and $100 \mu \mathrm{g} / \mathrm{mL}$ streptomycin in a humidified incubator with $5 \% \mathrm{CO}_{2}$ at $37^{\circ} \mathrm{C}$.

2.6. Grouping and Experimental Design. Human breast cancer nude mouse xenograft model was established by injecting MDA-MB-231 cell suspension $\left(1 \times 10^{7}\right.$ cells $\left./ \mathrm{mL}\right)$ from the left second pair breast pad in a volume of $0.5 \mathrm{~mL}$ each. Consequently, the oval skin bulge can be seen, with the nipple located at the center of the bulge. Tumors (visualized as a small nodule with an approximate size of $20-30 \mathrm{~mm}^{3}$ at the site of injection) appeared approximately 5-7 days after the injection. The mice were randomly and equally divided into three groups according to body weight and tumor size: triple-negative breast cancer control group (CON), tetrandrine group (TET), and capecitabine positive control group (Cap). The Cap group was intragastrically administered $0.1 \mathrm{~mL}$ of $10 \mathrm{mg} / \mathrm{mL}$ capecitabine solution, and the TET group was given $0.1 \mathrm{~mL}$ of $20 \mathrm{mg} / \mathrm{mL}$ TET solution, while the CON group was administered an equivalent volume of distilled water for 4 weeks. 
2.7. Tumor Measurement. The tumor growth and body weight of the mice were monitored on the $6^{\text {th }}, 12^{\text {th }}$, and $24^{\text {th }}$ day after intervention. The tumor volume was measured weekly by calipers until the mice were sacrificed under anesthesia. Each tumor was excised and weighed after the mice were sacrificed on day 29.

2.8. Sample Collection. After 4-week intervention, the mice were fasted for $24 \mathrm{~h}$, followed by sacrificing with $1 \%$ pentobarbital sodium $(0.1 \mathrm{mg} / \mathrm{g})$. The tumor tissue was excised and stripped. The tumor weight and volume were measured, after which one section of the tumor tissue was fixed in $4 \%$ paraformaldehyde solution for histopathology and one section was frozen in liquid nitrogen for further analysis.

2.9. Morphological Observation. Fresh tumor tissue samples were fixed in $4 \%$ paraformaldehyde. Then, the fixed tissues were embedded in paraffin, cut into $5 \mu \mathrm{m}$ slices, and stained with hematoxylin and eosin (HE), according to the standard protocol. The stained sections were observed and images acquired under a light microscope at 50×, 100×, and $400 \times$ magnification.

\subsection{Immunohistochemistry Analysis of Bcl-2-Associated $X$} Protein (BAX) and B-Cell Lymphoma/Leukemia-2 (BCL-2). The fresh tumor tissue samples were fixed in $4 \%$ paraformaldehyde and embedded in paraffin. $5 \mu \mathrm{m}$ thick paraffin sections were mounted on glass slides and treated with $3 \%$ hydrogen peroxide for $10 \mathrm{~min}$ at room temperature. After washing with phosphate-buffered saline (PBS), the sections were blocked with sheep serum for $10 \mathrm{~min}$ at room temperature, followed by incubation with anti-BAX or antiBCL-2 primary antibodies at $4^{\circ} \mathrm{C}$ overnight. The secondary antibodies were applied for $30 \mathrm{~min}$ at room temperature, and, then, the slides were stained with diaminobenzidine (DAB) and hematoxylin. The immunohistochemistry staining was observed under a microscope (BX-53; Olympus).

2.11. RNA Isolation and RT-qPCR. Total RNA was extracted using TRIzol reagent according to the manufacturer's instructions. The concentration and purity of the RNA samples were measured. cDNA was synthesized for reverse transcription-polymerase chain reaction (RT-PCR), according to the instructions in the First Strand cDNA Synthesis Kit (Invitrogen). The sequences of the primers used for the RTPCR assay are shown in Table 1 . The reverse transcription conditions were as follows: $37^{\circ} \mathrm{C}$ for $15 \mathrm{~min}$, followed by $85^{\circ} \mathrm{C}$ for $5 \mathrm{~s}$ for RT inactivation. The amplification was performed using the following conditions: $30 \mathrm{~s}$ at $95^{\circ} \mathrm{C}$ for denaturation, $5 \mathrm{~s}$ at $95^{\circ} \mathrm{C}$ for annealing, $40 \mathrm{~s}$ at $60^{\circ} \mathrm{C}$ for extension.

2.12. Western Blot Analysis. Frozen tumor tissues were homogenized in ice-cold RIPA lysis buffer (Beijing, China), and the extract was collected by centrifugation at $12000 \mathrm{rpm}$ for $15 \mathrm{~min}$ at $4^{\circ} \mathrm{C}$. The total protein concentration was determined by the bicinchoninic acid (BCA) protein assay kit (Beijing Pulilai Gene Technology Co., Ltd., Beijing, China). An equivalent amount of protein sample was separated by $12 \%$ sodium dodecyl sulfate polyacrylamide gel electrophoresis (SDS-PAGE) and transferred to a polyvinylidene difluoride (PVDF) membrane (Millipore, USA). Then, the membranes were blocked at room temperature with $5 \%$ dried skimmed milk for $1 \mathrm{~h}$ and incubated at $4^{\circ} \mathrm{C}$ overnight with primary antibodies: Caspase-3, Survivin, Bax, $\mathrm{Bcl}-2$, Bid, PARP, and $\beta$-actin. The washed membranes were incubated with the corresponding secondary antibodies for $1 \mathrm{~h}$ at room temperature. Finally, the immunoreactive bands were detected using an ECL detection system (Tanon, Shanghai, China) and quantified using Image J software.

2.13. Statistical Analysis. Statistical analysis was performed using SPSS 17.0 statistical software (IBM, Armonk, NY, USA). Data were presented as mean \pm standard deviation (SD). Significant differences among groups were analyzed by one-way analysis of variance (ANOVA), followed by leastsquare difference test. $P<0.05$ was considered statistically significant.

\section{Results}

3.1. Effect of TET on Body Weight. With the experiment prolonged duration, compared to the control group, the other groups of nude mice showed weight loss, slow response, arched neck, and prominent spine. The body weights of nude mice at different time points are shown in Table 2.

3.2. Effect on Tumor Weight and Volume. The tumor volume of each group of nude mice increased with time, establishing a positive correlation between the two parameters.

After 24-day intervention, the tumor weight in the TET and Cap groups decreased significantly as compared to the control group $(P<0.05)$. Compared to the TET group, the tumor weight in the Cap group decreased significantly $(P<0.05)$ (Table 3$)$.

Compared to the control group, tumor weight and volume in both TET and Cap groups decreased significantly $(P<0.05)$. Compared to the TET group, the tumor weight and volume in the Cap group decreased significantly $(P<0.05$; Table 4, Figure 1).

3.3. Histopathological Changes in the Tumor Tissue. The cells of tumor tissues in all the groups were disorderly arranged and nested, with different morphologies and enlarged nuclei. Compared to the control group, different levels of inflammatory cell aggregates were distributed in a sheet form in the TET and Cap groups. In the Cap group, the nuclei were almost invisible and stained with pink, indicating cell rupture and cytoplasmic outflow. In the TET group, different degrees of pink cytoplasmic staining and mixed purple-blue nuclear staining were observed. Compared to the control group, the pink cytoplasm staining area in both TET and Cap 
TABLE 1: Primer sequences of RT-qPCR.

\begin{tabular}{lrc}
\hline Gene & Upstream primer & Downstream primer \\
\hline Caspase-3 & $5^{\prime}$-GGAGGCCGACTTCTTGTATG-3' \\
Bid & $5^{\prime}$-GGCCTACCCTAGAGACATGGA-3' & $5^{\prime}$-ACTGTTTCAGCATGGCACAA-3' \\
Bax & $5^{\prime}$-ATGGGCTGGACATTGGAC-3' & $5^{\prime}$-AGACATCACGGAGCAAGGAC-3' \\
Bcl-2 & $5^{\prime}$-ATGTGTGTGGAGAGCGTCAA-3' & $5^{\prime}$-GGGACATCAGTCGCTTCAG-3' \\
Survivin & $5^{\prime}$-GGACCACCGCATCTCTACAT-3' & $5^{\prime}$-GAGACAGCCAGGAGAAATCAA-3' $^{\prime}$ \\
PARP & $5^{\prime}$-CCGCATACTCCATCCTCAGT-3' & $5^{\prime}$-CAAGTCTGGCTCGTTCTCAGT-3' \\
GAPDH & $5^{\prime}$-AGAAGGCTGGGGCTCATTTG-3' & $5^{\prime}$-GCTTCTTCATCCCAAAGTCG-3' \\
\hline
\end{tabular}

TABLe 2: Body weight of nude mice at different time points (mean $\pm \mathrm{SD}$ ).

\begin{tabular}{lccccc}
\hline Groups & $N$ & Day 0 $(\mathrm{g})$ & Day 6 $(\mathrm{g})$ & Day 12 $(\mathrm{g})$ & Day 24 $(\mathrm{g})$ \\
\hline CON & 10 & $14.56 \pm 0.33$ & $15.47 \pm 0.26$ & $16.92 \pm 0.12$ & $18.02 \pm 0.27$ \\
TET & 10 & $14.64 \pm 0.45$ & $15.06 \pm 0.12$ & $15.13 \pm 0.21^{*}$ & $15.28 \pm 0.30^{*}$ \\
Cap & 10 & $14.53 \pm 0.45$ & $15.15 \pm 0.26$ & $15.13 \pm 0.20^{*}$ & $15.47 \pm 0.50^{*}$ \\
\hline
\end{tabular}

Note: *compared to the control group, $P<0.05$.

TABLE 3: Tumor volume at different time points (mean \pm SD) $(n=10)$.

\begin{tabular}{lccccc}
\hline Groups & $N$ & D0 $\left(\mathrm{cm}^{3}\right)$ & D6 $\left(\mathrm{cm}^{3}\right)$ & D12 $\left(\mathrm{cm}^{3}\right)$ & D24 $\left(\mathrm{cm}^{3}\right)$ \\
\hline CON & 10 & $0.04 \pm 0.01$ & $0.84 \pm 0.06$ & $1.57 \pm 0.03$ & $2.50 \pm 0.02$ \\
TET & 10 & $0.04 \pm 0.01$ & $0.77 \pm 0.05$ & $1.33 \pm 0.04^{*}$ & $1.85 \pm 0.04^{*}$ \\
Cap & 10 & $0.04 \pm 0.01$ & $0.69 \pm 0.02^{*}$ & $1.36 \pm 0.03^{*}$ & $1.37 \pm 0.03^{* \#}$ \\
\hline
\end{tabular}

Note: ${ }^{*}$ compared to the control group, $P<0.05 ;{ }^{\#}$ compared to the TET group, $P<0.05$.

TABLE 4: Tumor weight and volume after excision (mean \pm SD) $(n=10)$.

\begin{tabular}{lccc}
\hline Groups & $N$ & Weight $(\mathrm{g})$ & Volume $\left(\mathrm{cm}^{3}\right)$ \\
\hline CON & 10 & $3.28 \pm 0.05$ & $2.62 \pm 0.03$ \\
TET & 10 & $2.65 \pm 0.03^{*}$ & $2.01 \pm 0.03^{*}$ \\
Cap & 10 & $1.92 \pm 0.04^{*} \#$ & $1.44 \pm 0.03^{*}$ \\
\hline
\end{tabular}

Note: * compared to the control group, $P<0.05 ;{ }^{\#}$ compared to the TET group, $P<0.05$.

groups was larger, indicating a high proportion of cell necrosis (Figure 2).

3.4. Immunohistochemistry Analysis of Bcl-2 and Bax. Compared to the control group, the expression of Bcl-2 decreased significantly in the TET and Cap groups. The decreased expression of Bcl-2 in the Cap group was superior to that in the TET group.

Compared to the control group, the expression of Bax was increased in both TET and Cap groups; interestingly, the increased expression in the Cap group was superior to that in the TET group (Figure 3).

3.5. Gene Expression. Compared to the control group, the expression of Caspase-3, Bax, Bid, and PARP increased significantly in the TET and Cap groups $(P<0.05,0.01)$, while the expression of Bcl-2 and Survivin in the TET and Cap groups decreased significantly $(P<0.05)$. Compared to the TET group, the expression of Caspase-3, Bax, Bid, and PARP increased significantly in the Cap group $(P<0.05$, 0.01 , while the expression of Survivin decreased significantly $(P<0.05)$ (Figure 4$)$.

3.6. Protein Expression. Compared to the control group, the expression of Caspase-3, Bax, and Bid proteins increased significantly in both TET and Cap groups $(P<0.05,0.01)$, while that of Bcl-2, Survivin, and PARP decreased significantly in both TET and Cap groups $(P<0.05,0.01)$. Compared to the TET group, the protein expression of Caspase-3, Bax, and Bid proteins increased significantly in the Cap group $(P<0.01)$ (Figure 5$)$.

\section{Discussion}

In this study, the anticancer activity of TET against the TNBC cell line MDA-MB-231 in vivo was investigated by focusing on apoptosis. In addition to morphological change, the expression of apoptosis-associated genes, such as Caspase-3, Survivin, Bax, Bcl-2, Bid, and PARP was detected.

The present study demonstrated that TET markedly inhibits the growth of tumors in MDA-MB-231 mouse xenografts, as the tumor weight and volume decreased significantly in the TET and Cap groups. This finding was consistent with that of Zhang et al., who demonstrated that TET effectively suppressed the tumor growth in cervical cancer [25]. The morphological analysis demonstrated that the tumor cells were arranged in a disorderly, irregular, nested shape, with different sizes and shapes, and enlarged nucleus, as observed in the TNBC control group. In TET and Cap groups, inflammatory cells accumulated due to cell necrosis. These results suggested that TET exerted notable antitumor effects on TNBC cell line MDA-MB-231 in vivo.

Apoptosis plays a major role in killing tumor cells [25]. It is a gene-regulated cell death process, also known as programmed cell death, which occurs in all living cells and is regulated by genes, characterized by a series of cellular morphological changes, including chromosome condensation, nuclear fragmentation, cell shrinkage, and the 

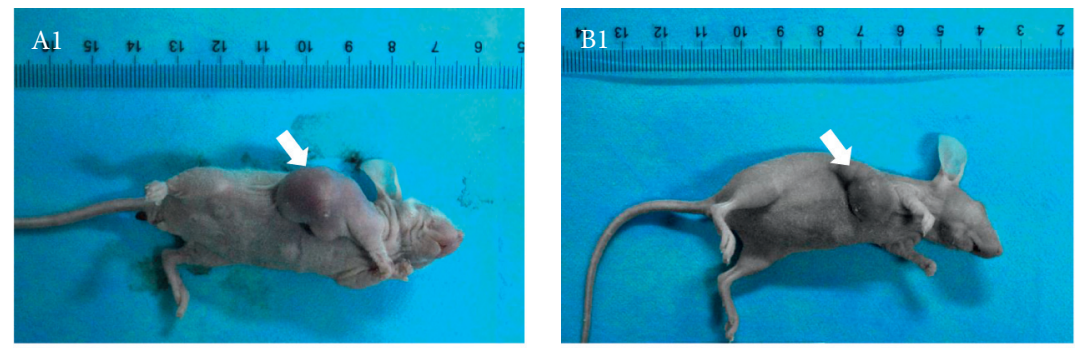

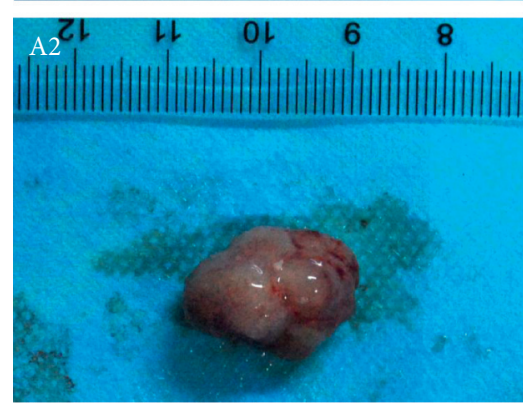

(a)

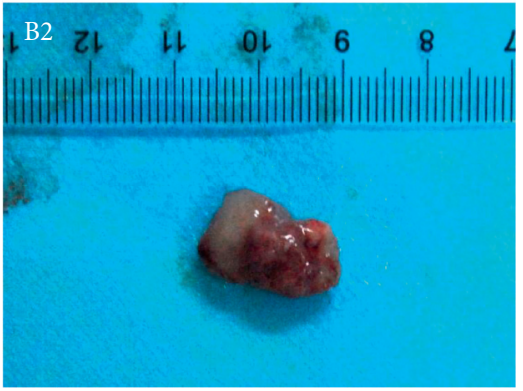

(b)
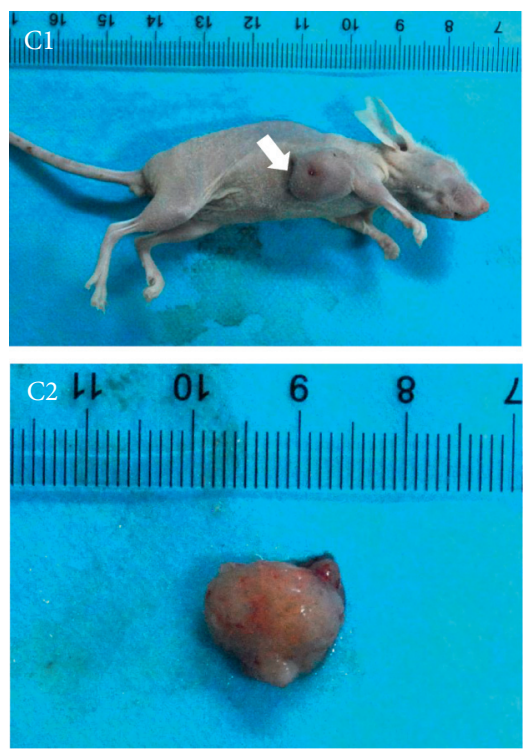

(c)

FIGURE 1: Antitumor activity of TET and Cap in MDA-MB-231 mouse xenografts. Photographs of tumor appearance (A1: control group; B1: TET group; C1: Cap group). Photographs of isolated tumors derived from control and treated mice (A2: control group; B2: TET group; C2: Cap group).

formation of apoptosome [21]. The extrinsic and intrinsic signaling pathways lead to apoptosis. The extrinsic pathway provokes apoptosis through a caspase cascade that eventually leads to cell death, while the intrinsic apoptotic pathway is mitochondria-dependent and responds to different stress conditions, such as cytosolic calcium, genetic damage, and oxidative stress [33]. Both pathways triggered caspase cascade, converged on Caspase-3, and ultimately led to the morphological characteristics of apoptosis [24].

The Bcl-2 family is involved in the regulation of cell apoptosis, including a series of antiapoptotic and proapoptotic members. The Bcl-2 apoptotic signaling pathway activates the apoptotic pathway through multiple intercellular and intracellular signals. Within the Bcl family, Bax is a proapoptotic protein, which promotes apoptosis by activating caspases. Conversely, $\mathrm{Bcl}-2$ is an antiapoptotic protein, which prevents apoptosis by inhibiting the release of mitochondrial apoptogenic factors into the cytoplasm. Bcl-2 and Bax are typical proteins that inhibit and promote apoptosis, respectively, in this pathway. Apoptosis is mainly determined by the relative concentration of $\mathrm{Bcl}-2$ and Bax. An imbalance of Bax and Bcl-2 proteins may lead to the loss of MMP and the release of cytochrome $\mathrm{C}$, which activates Caspase-3 and results in apoptosis [21, 34-37]. The BID protein is a member of another group of the Bcl-2 family. It activates apoptosis as well as integrating two main apoptotic 


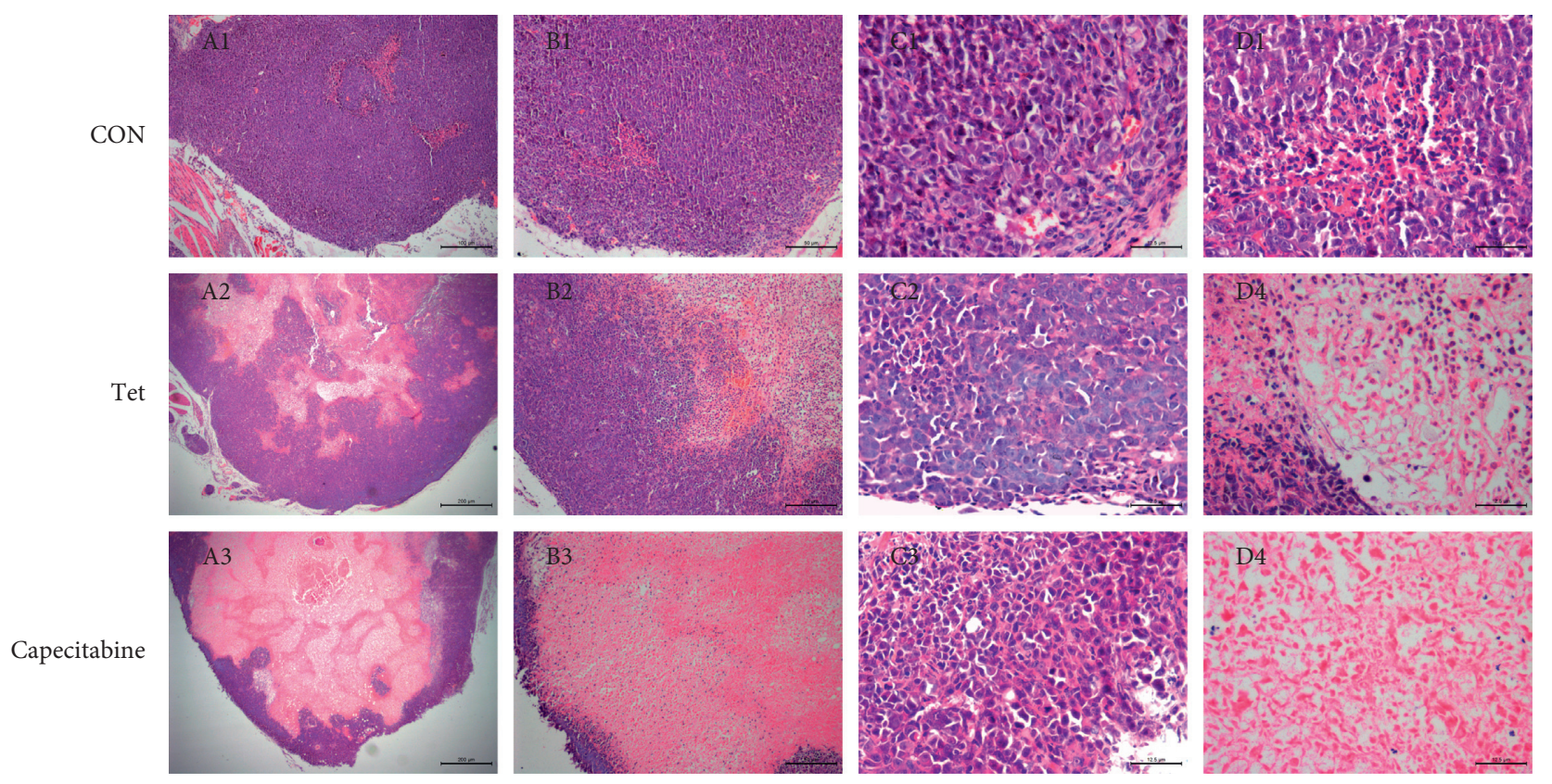

Figure 2: Morphological changes of tumor tissue in the CON, TET, and cap groups. A represents tumor full-section at 50× magnification; B represents tumor junction-section at $100 \times$ magnification; $\mathrm{C}$ represents tumor marginal area at $400 \times$ magnification; and D represents tumor core region at $400 \times$ magnification.
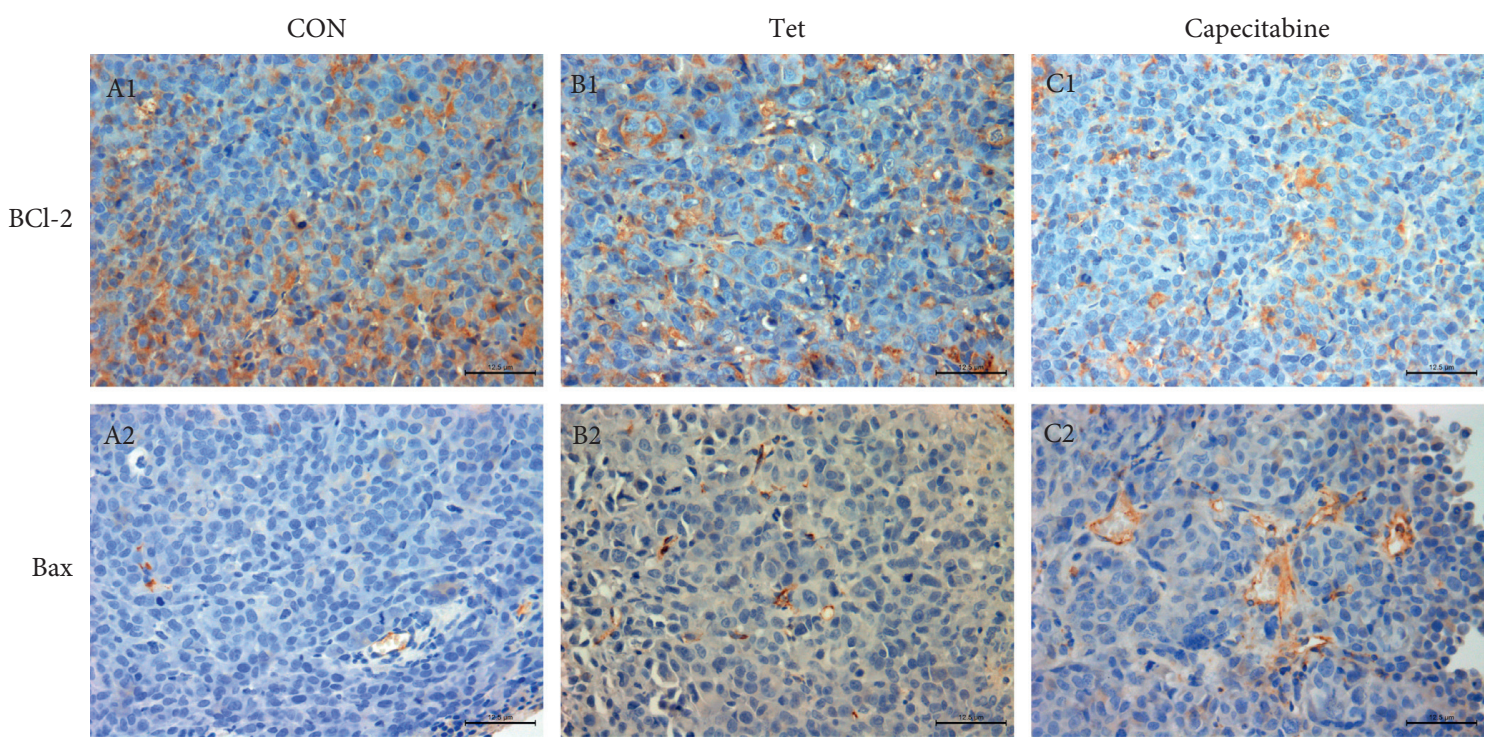

Figure 3: TET induces cell apoptosis in TNBC cell line MDA-MB-231 xenografts. A represents CON group; B represents TET group; and C represents cap group. The expression of Bcl-2 and Bax in tumors was detected by immunohistochemistry (400× magnification).

routes, connecting the membranous (external) and mitochondrial (internal) pathways [38]. In this study, the immunohistochemistry analysis showed decreased expression of Bcl-2 in the TET and Cap groups as compared to the CON group; however, the decreased expression of $\mathrm{Bcl}-2$ in the Cap group was superior to that in the TET group. Compared to the control group, the expression of Bax was increased in both the TET and Cap groups, while the increased expression in the Cap group was superior to that in the TET group. RT-PCR and Western blotting results showed that
Tet decreased the level of antiapoptotic gene and protein $\mathrm{Bcl}$ 2 and increased the level of proapoptotic genes and proteins, $\mathrm{Bax}$ and Bid. Considering that these three proteins are closely associated with mitochondria, it could be speculated that Tet induced apoptosis through mitochondrial disruption by changing the levels of the apoptosis-associated proteins, Bcl-2, Bax, and Bid.

Caspase- 3 plays a major role in triggering the apoptotic process, and its activity has been suggested to be an index of apoptosis, which is commonly considered as the most 


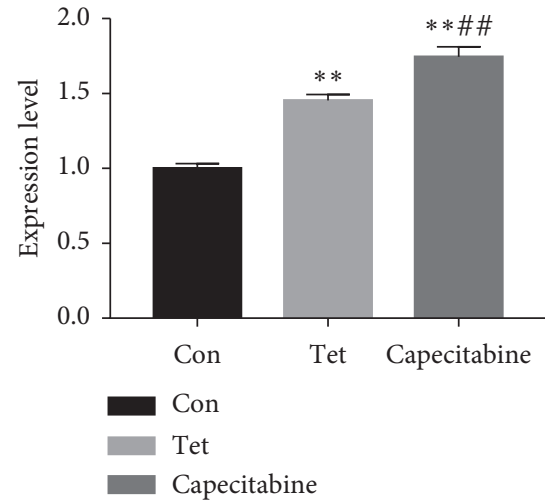

(a)

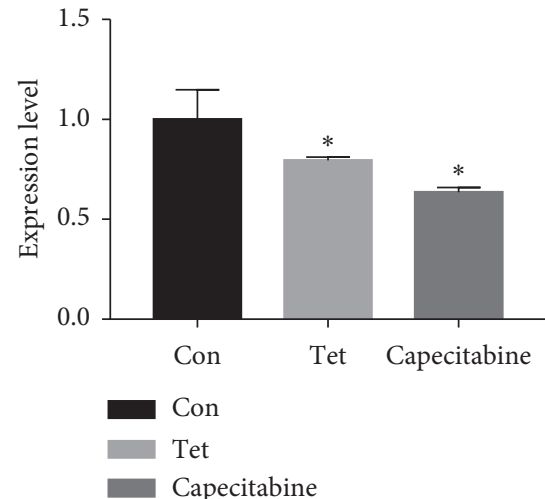

(d)

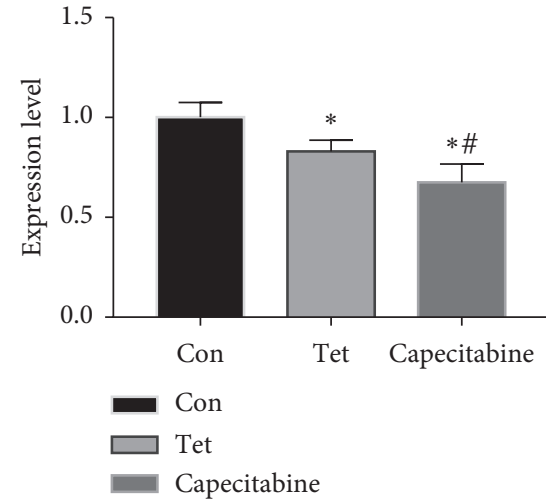

(b)

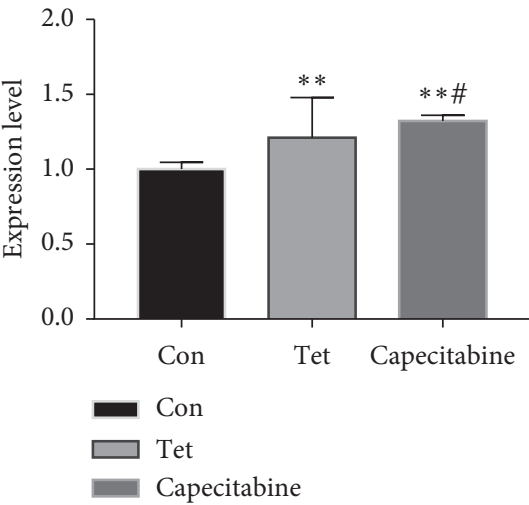

(e)

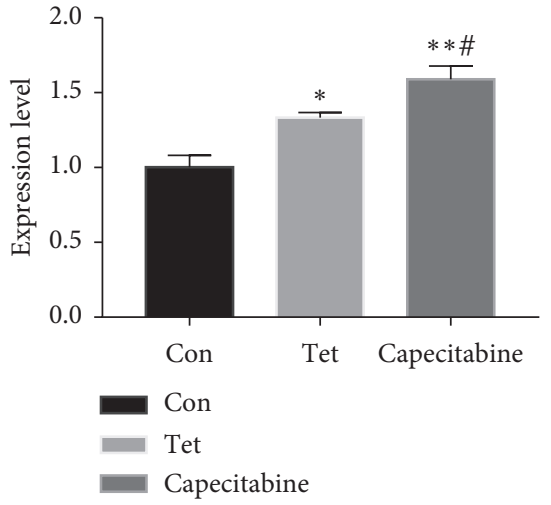

(c)

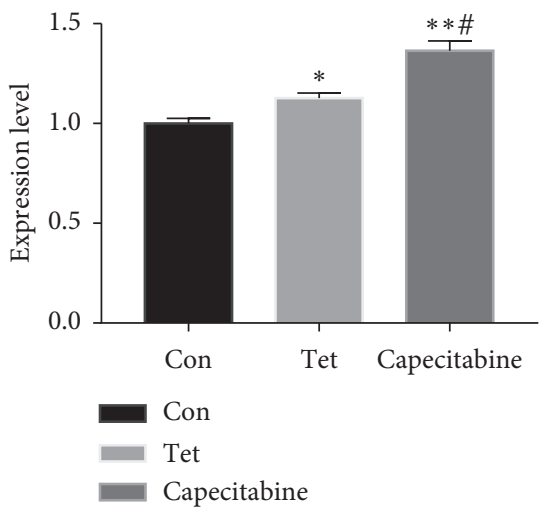

(f)

FIGURE 4: Effect of TET on apoptosis-associated gene expression in the breast tumor as determined by RT-qPCR. The levels of (a) Caspase-3, (b) Survivin, (c) Bax, (d) Bcl-2, (e) Bid, and (f) PARP genes in tumors were determined by RT-qPCR. *Compared to the control group, $P<0.05 ;{ }^{* *}$ compared to the control group, $P<0.01$; ${ }^{*}$ compared to the TET group, $P<0.05$; ${ }^{\#}$ compared to the TET group, $P<0.01$.

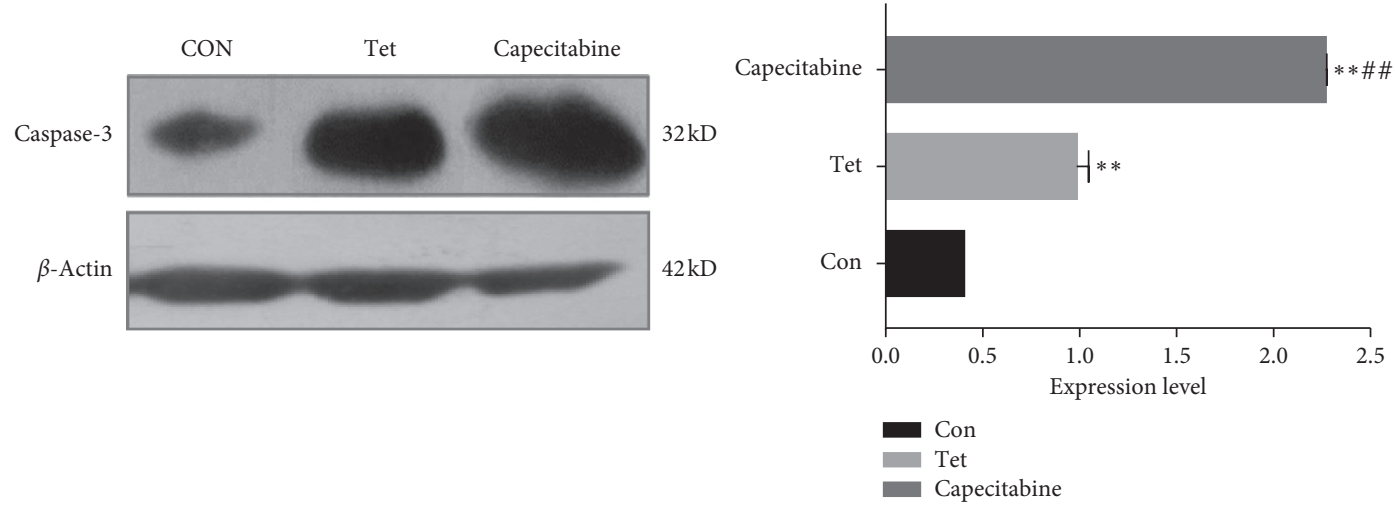

(a)

FIgURE 5: Continued. 


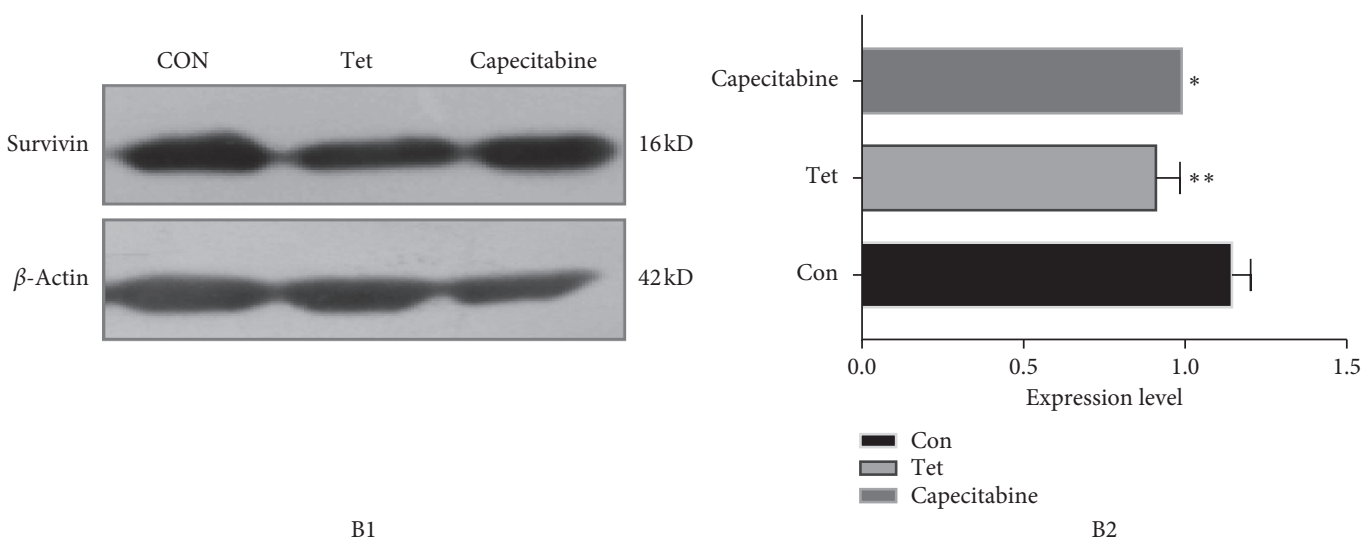

(b)
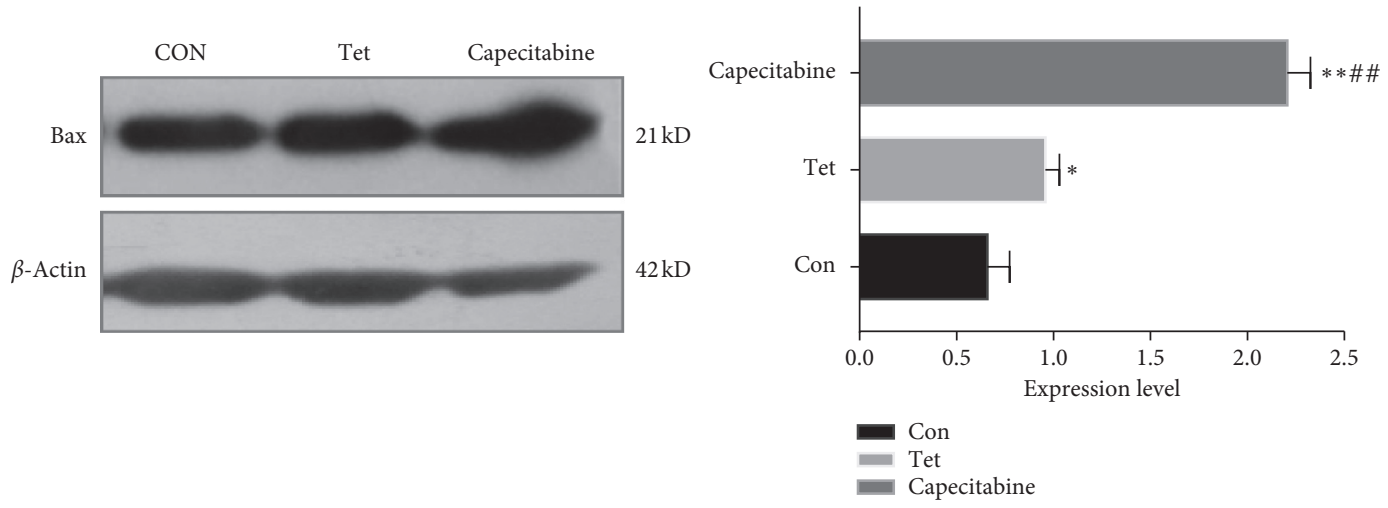

C1

(c)
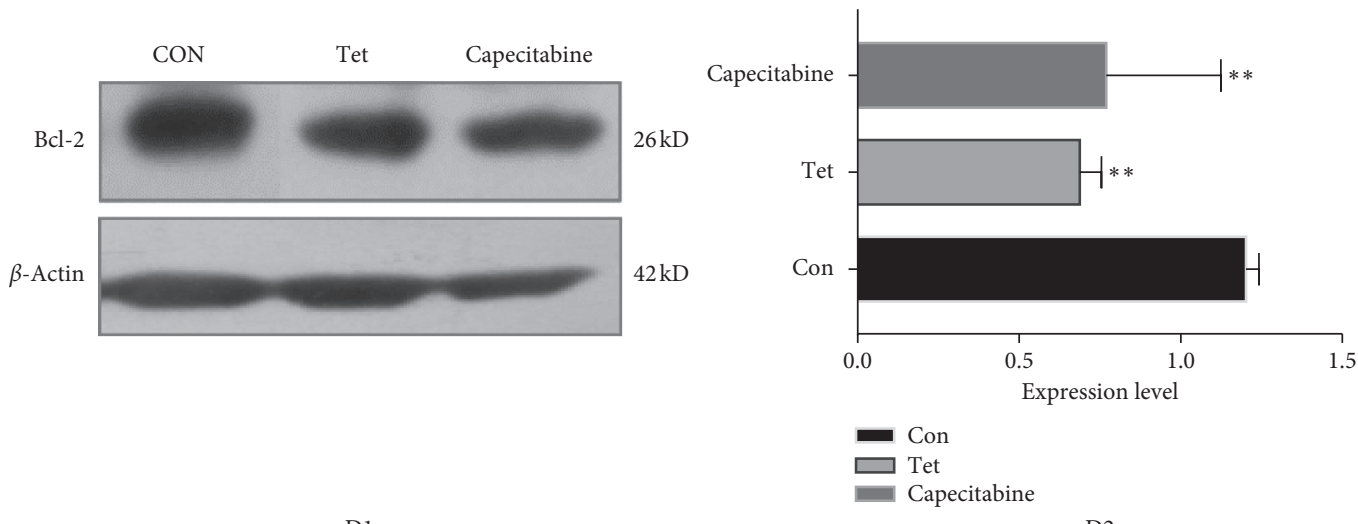

D1

(d)

Figure 5: Continued. 

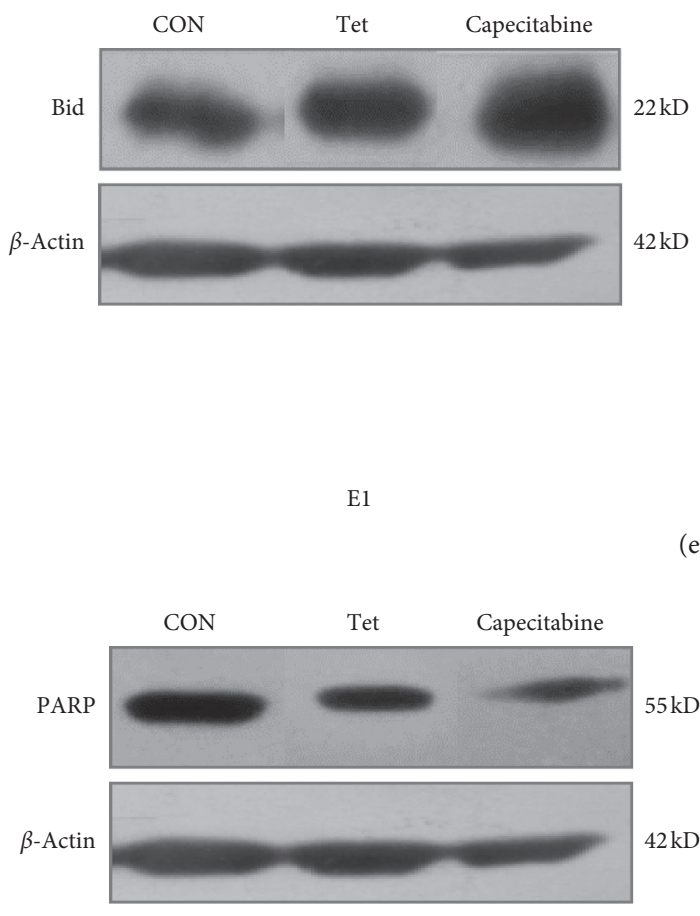

F1

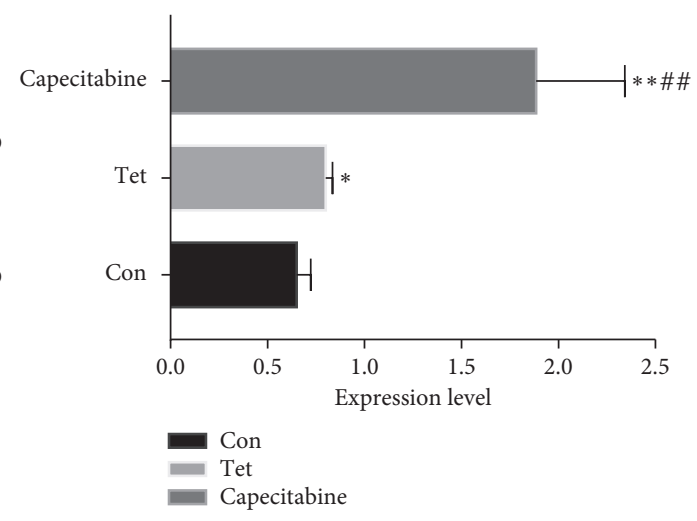

E2

(e)

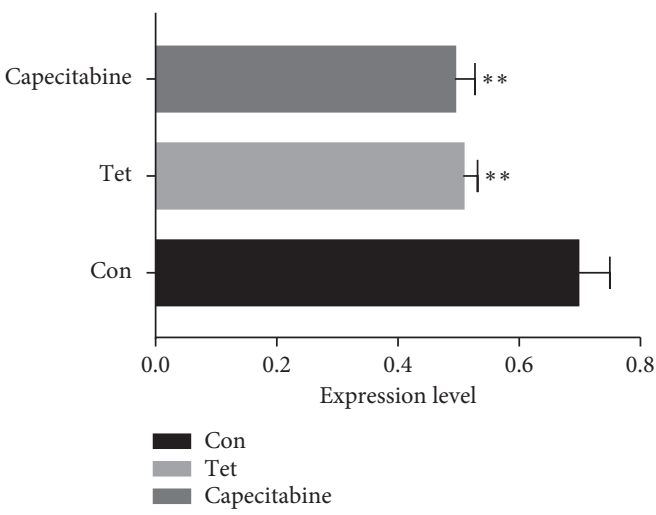

F2

(f)

Figure 5: Effect of TET on apoptosis-associated protein expression in the breast tumor as determined by Western blotting. The protein levels of (a) Caspase-3, (b) Survivin, (c) Bax, (d) Bcl-2, (e) Bid, and (f) PARP in tumors were determined by Western blotting. A represents expression of caspase 3; B represents expression of Survivin; $C$ represents expression of Bax; D represents expression of Bcl-2; E represents expression of Bid; F represents expression of PARP. ${ }^{*}$ Compared to the control group, $P<0.05 ;{ }^{* *}$ compared to the control group, $P<0.01$; \#\# compared to the TET group, $P<0.01$.

important terminal cleavage enzyme during apoptosis. PARP is involved in DNA repair and transcriptional regulation and is regarded as a critical regulatory factor of cell survival and cell death. It also participates in the regulation of transcription factors in tumorigenesis and inflammatory response [39]. During apoptosis, PARP is cleaved by Caspase- 3 into two fragments of $31 \mathrm{kDa}$ and $85 \mathrm{kDa}$. This cleavage makes PARP unable to function normally, causing apoptosis [40]. Survivin belongs to the inhibitor of apoptosis protein (IAP) family and is potentially involved in both facilitating tumor cell proliferation and inhibiting apoptosis. It is lowly expressed in normal cells, but highly during tumor proliferation and angiogenesis. Tumor apoptosis can be induced by inhibiting this protein [41]. It has been shown that Survivin can directly block the process and activation of the cell death terminal effector Caspase-3 and induce the apoptosis of cells [37]. In this study, compared to the control group, the expression of Caspase- 3 gene and protein increased significantly in both TET and Cap groups, while that of Survivin and PARP decreased significantly in both groups. These results demonstrated that TET induces apoptosis by the activation of Caspase-3, cleavage of PARP, and downregulation of Survivin in TNBC in vivo.

\section{Conclusion}

In this study, we demonstrated that TET effectively inhibits tumor growth and induces apoptosis in TNBC cell line MDA-MB-231. The mechanism underlying this effect may be mediated by TET-upregulated Caspase-3, Bax, and Bid, and downregulated Bcl-2, Survivin, and PARP. This study showed that TET is a promising therapeutic agent for the treatment of TNBC. Considering TNBC is one of the most difficult subtypes of breast cancer to treat due to its aggressive, metastatic behavior and a lack of targeted therapy, further studies about the effect of TET on the chemotherapeutic resistance are imperative.

\section{Data Availability}

The data supporting the conclusions of this study are available to all interested readers upon request to the corresponding author(pxh_127@163.com). 


\section{Conflicts of Interest}

The authors declare no conflicts of interest.

\section{Authors' Contributions}

Xiao-hua pei carried out the research and designed the experiment. Chun-hui Wang and Jiamin Yang contributed to data analysis and manuscript writing. Chunhui Wang, Yubo Guo, Jing Shen, and Jiamin Yang contributed to experimental manipulation and data acquisition. Chun-hui Wang and Jia-min Yang contributed equally to this work.

\section{Acknowledgments}

This work was supported by the Beijing Municipal Natural Science Foundation (Grant no. 7172127) and the National Natural Science Foundation of China (Grant no. 81560775).

\section{References}

[1] O. Beiki, P. Hall, A. Ekbom, and T. Moradi, "Breast cancer incidence and case fatality among 4.7 million women in relation to social and ethnic background: a population-based cohort study," Breast Cancer Research, vol. 14, no. 1, p. 13, 2012.

[2] R. L. Siegel, K. D. Miller, and A. Jemal, "Cancer statistics, 2016," CA: A Cancer Journal for Clinicians, vol. 66, no. 1, pp. 7-30, 2016.

[3] L. Fan, K. Strasser-Weippl, J.-J. Li et al., "Breast cancer in China," The Lancet Oncology, vol. 15, no. 7, pp. e279-e289, 2014.

[4] Z. H. Li, Y. R. Qiu, W. Q. Lu, Y. Jiang, and J. Wang, "Immunotherapeutic interventions of triple negative breast cancer," Journal of Translational Medicine, vol. 16, no. 1, p. 19, 2018.

[5] A. Gucalp and T. A. Traina, "Targeting the androgen receptor in triple-negative breast cancer," Current Problems in Cancer, vol. 40, no. 2-4, pp. 141-150, 2016.

[6] J. M. Lebert, R. Lester, E. Powell, M. Seal, and J. McCarthy, "Advances in the systemic treatment of triple-negative breast cancer," Current Oncology, vol. 25, no. 1, pp. 142-150, 2018.

[7] J.-R. Jhan and E. R. Andrechek, "Triple-negative breast cancer and the potential for targeted therapy," Pharmacogenomics, vol. 18, no. 17, pp. 1595-1609, 2017.

[8] V. J. Shine, G. I. Anuja, S. R. Suja, G. Raj, and P. G. Latha, "Bioassay guided fractionation of Cyclea peltata using in vitro RAW 264.7 cell culture, antioxidant assays and isolation of bioactive compound tetrandrine," Journal of Ayurveda and Integrative Medicine, p. 6, 2018.

[9] F. Wang, J. Wang, Z. Zhang, and S. Chen, "Tetrandrine inhibits the proliferation and cytokine production induced by IL-22 in HaCaT cells," Journal of International Medical Research, vol. 46, no. 12, pp. 5210-5218, 2018.

[10] W. Xie and L. Du, "Diabetes is an inflammatory disease: evidence from traditional Chinese medicines," Diabetes, Obesity and Metabolism, vol. 13, no. 4, pp. 289-301, 2011.

[11] H. Yao, Z. Cao, L. Peng et al., "A novel controlled release tetrandrine-loaded PDLLA film: evaluation of drug release and anti-adhesion effects in vitro and in vivo," Drug Delivery and Translational Research, vol. 10, no. 1, pp. 13-22, 2020.

[12] Y.-C. Hsu, Y.-T. Chiu, C.-C. Cheng, C.-F. Wu, Y.-L. Lin, and Y.-T. Huang, "Antifibrotic effects of tetrandrine on hepatic stellate cells and rats with liver fibrosis," Journal of Gastroenterology and Hepatology, vol. 22, no. 1, pp. 99-111, 2007.

[13] B. Yu, B. Yuan, A. Kiyomi et al., "Differentiation induction of human breast cancer cells by arsenite in combination with tetrandrine," American Journal of Translational Research, vol. 11, no. 12, pp. 7310-7323, 2019.

[14] D. E. Kim, J. S. Min, M. S. Jang et al., "Natural bis-benzylisoquinoline alkaloids-tetrandrine, fangchinoline, and cepharanthine, inhibit human coronavirus OC43 infection of MRC-5 human lung cells," Biomolecules, vol. 9, no. 11, 2019.

[15] X. Wang, Y. Yang, D. Yang et al., "Tetrandrine prevents monocrotaline-induced pulmonary arterial hypertension in rats through regulation of the protein expression of inducible nitric oxide synthase and cyclic guanosine monophosphatedependent protein kinase type 1," Journal of Vascular Surgery, vol. 64, no. 5, pp. 1468-1477, 2016.

[16] J. Sun, P. Song, Y. Wang, and Y. Chen, "Clinical efficacy of acetylcysteine combined with tetrandrine tablets in the treatment of silicosis and the effect on serum IL- 6 and TNF$\alpha$," Experimental and Therapeutic Medicine, vol. 18, no. 5, pp. 3383-3388, 2019.

[17] D. Liao, W. Zhang, P. Gupta et al., "Tetrandrine interaction with $\mathrm{ABCB} 1$ reverses multidrug resistance in cancer cells through competition with anti-cancer drugs followed by downregulation of ABCB1 expression," Molecules, vol. 24, no. 23, p. 18, 2019.

[18] Y. B. Guo and X. H. Pei, "Tetrandrine-induced autophagy in MDA-MB-231 triple-negative breast cancer cell through the inhibition of PI3K/AKT/mTOR signaling," Evidence-Based Complementary and Alternative Medicine, vol. 2019, Article ID 7517431, 11 pages, 2019.

[19] C. Liang, X. Pei, and X. H. Pei, "Tetrandrine and arsenic trioxide synergistically inhibit proliferation of HCC1937 triple negative breast cancer cells," Journal of Traditional Chinese Medicine, vol. 37, no. 4, pp. 436-443, 2017.

[20] Z. X. Zhang, T. Liu, M. Yu, K. D. Li, and W. H. Li, “The plant alkaloid tetrandrine inhibits metastasis via autophagy-dependent $\mathrm{Wnt} / \beta$-catenin and metastatic tumor antigen 1 signaling in human liver cancer cells," Journal of Experimental \&clinical Cancer Research, vol. 37, no. 1, p. 11, 2018.

[21] J. N. Li, Q. H. Wang, Z. B. Wang et al., "Tetrandrine inhibits colon carcinoma HT-29 cells growth via the Bcl-2/Caspase 3/ PARP pathway and G1/S phase," Bioscience Reports, vol. 39, no. 5, p. 12, 2019.

[22] G. X. Wu, T. Liu, H. Li, Y. F. Li, D. J. Li, and W. H. Li, “c-MYC and reactive oxygen species play roles in tetrandrine-induced leukemia differentiation," Cell Death \& Disease, vol. 9, no. 5, p. 16, 2018.

[23] Z. Chen, L. Zhao, F. Zhao, G. Yang, and J. J. Wang, “Tetrandrine suppresses lung cancer growth and induces apoptosis, potentially via the VEGF/HIF- $1 \alpha$ /ICAM- 1 signaling pathway," Oncology Letters, vol. 15, no. 5, pp. 7433-7437, 2018.

[24] W. Liu, B. Kou, Z. K. Ma et al., "Tetrandrine suppresses proliferation, induces apoptosis, and inhibits migration and invasion in human prostate cancer cells," Asian Journal of Andrology, vol. 17, no. 5, pp. 850-853, 2015.

[25] H. Y. Zhang, B. B. Xie, Z. Zhang, X. G. Sheng, and S. Q. Zhang, "Tetrandrine suppresses cervical cancer growth by inducing apoptosis in vitro and in vivo," Drug Design, Development and Therapy, vol. 13, pp. 119-127, 2019.

[26] Y. L Dang, Y. S. Xu, W. T. Wu et al., "Tetrandrine suppresses lipopolysaccharide-induced microglial activation by inhibiting NF- $\kappa \mathrm{B}$ and ERK signaling pathways in BV2 cells," PLoS One, vol. 9, no. 8, Article ID e102522, 2014. 
[27] T. K. Juan, K. C. Liu, C. L. Kuo et al., “Tetrandrine suppresses adhesion, migration and invasion of human colon cancer SW620 cells via inhibition of nuclear factor- $\kappa \mathrm{B}$, matrix metalloproteinase-2 and matrix metalloproteinase-9 signaling pathways," Oncology Letters, vol. 15, no. 5, pp. 7716-7724, 2018.

[28] W. C. Lin, W. H. Wang, Y. H. Lin et al., "Synergistic effects of tetrandrine combined with ionizing radiation on a murine colorectal carcinoma-bearing mouse model," Oncology Reports, vol. 40, no. 3, pp. 1390-1400, 2018.

[29] X. Sun, R. Xu, Y. Deng et al., "Effects of tetrandrine on apoptosis and radiosensitivity of nasopharyngeal carcinoma cell line CNE," Acta biochimica et biophysica Sinica, vol. 39, no. 11, pp. 869-878, 2007.

[30] Y. Hou, T. Guo, C. Wu, and X. He, "Effect of tetrandrine combined with epirubicin on the growth of human breast carcinoma multidrug resistance cell line," Yakugaku Zasshi, vol. 128 , no. 4, pp. 663-666, 2008.

[31] W. C. Xu, X. Q. Wang, Y. C. Tu et al., "Tetrandrine and cepharanthine induce apoptosis through caspase cascade regulation, cell cycle arrest, MAPK activation and PI3K/Akt/ mTOR signal modification in glucocorticoid resistant human leukemia Jurkat T cells," Chemico-biological Interactions, vol. 310, 2019.

[32] J. H. Huang, X. H. Huang, Z. Y. Chen, Q. S. Zheng, and R. Y. Sun, "Dose conversion among different animals and healthy volunteers in pharmacological study," Chinese Journal of Clinical Pharmacology and Therapeutics, vol. 9, no. 9, pp. 1069-1072, 2004.

[33] D. K. Jang, I. S. Lee, H. S. Shin, and H. M. Yoo, " $2 \alpha-$ Hydroxyeudesma-4,11(13)-Dien-8 $\beta, 12$-Olide isolated from inula britannica induces apoptosis in diffuse large B-cell lymphoma cells," Biomolecules, vol. 10, no. 2, p. 16, 2020.

[34] J. M. Adams and S. Cory, "The Bcl-2 apoptotic switch in cancer development and therapy," Oncogene, vol. 26, no. 9, pp. 1324-1337, 2007.

[35] L. E. Jonges, J. F. Nagelkerke, N. G. Ensink et al., "Caspase-3 activity as a prognostic factor in colorectal carcinoma," Laboratory Investigation, vol. 81, no. 5, pp. 681-688, 2001.

[36] F. Llambi and D. R. Green, "Apoptosis and oncogenesis: give and take in the BCL-2 family," Current Opinion in Genetics \& Development, vol. 21, no. 1, pp. 12-20, 2011.

[37] H. Liang, M. N. Gu, C. X. Yang, H. B. Wang, X. J. Wen, and Q. L. Zhou, "Sevoflurane inhibits proliferation, induces apoptosis, and blocks cell cycle progression of lung carcinoma cells," Asian Pacific Journal of Cancer Prevention: APJCP, vol. 12, no. 12, pp. 3415-3420, 2011.

[38] M. Gryko, A. Pryczynicz, K. Zareba, B. Kędra, A. Kemona, and K. G. Ustymowicz, "The expression of Bcl-2 and BID in gastric cancer cells," Journal of Immunology Research, vol. 2014, Article ID 953203, 5 pages, 2014.

[39] X. Min, H. Heng, H. L. Yu et al., "Anticancer effects of 10hydroxycamptothecin induce apoptosis of human osteosarcoma through activating caspase-3, p53 and cytochrome c pathways," Oncology Letters, vol. 15, no. 2, pp. 2459-2464, 2018.

[40] J. Song, J. Lan, C. Chen et al., "Design, synthesis and bioactivity investigation of tetrandrine derivatives as potential anti-cancer agents," MedChemComm, vol. 9, no. 7, pp. 1131-1141, 2018.

[41] X.-Q. Chen, S. Yang, M.-Q. Kang, Z.-Y. Li, H.-S. Lu, and T.-Y. Lin, "Survivin expression in human lung cancer and the influence of its downregulation on the biological behavior of human lung cancer cells," Experimental and Therapeutic Medicine, vol. 3, no. 6, pp. 1010-1014, 2012. 